\title{
IS MULLIGAN'S SUSTAINED NATURAL APOPHYSEAL GLIDES (SNAGS) OR MUSCLE ENERGY TECHNIQUE IS EFFECTIVE IN THE NON-SURGICAL MANAGEMENT OF CERVICOGENIC HEADACHE? A TWO-GROUP PRETEST-POSTTEST RANDOMIZED CONTROLLED TRIAL
}

\author{
VEENA KIRTHIKA $S^{*}$, PADMANABHAN K, SELVARAJ SUDHAKAR, VIJAYA KUMAR M
}

Faculty of Physiotherapy, Dr. M.G.R Educational And Research Institute, Maduravoyal, Chennai - 600 095, Tamil Nadu, India. Email: veena. physio@drmgrdu.ac.in

Received: 20 April 2018, Revised and Accepted: 23 May 2018

ABSTRACT

Objective: The purpose of this study is to compare the efficacy of Mulligan's Glides like sustained natural apophyseal glides and muscle energy technique (MET) in the management of individual with cervicogenic headache (ICH).

Methods: A total of 30 ICH were recruited by the simple random sampling to participate in this two-group pretest-posttest, single-blinded randomized clinical study. Recruited ICH was randomly allocated into two groups, Group A and Group B. ICH in Group A was provided with Mulligan's SNAGs of 3 glides/session/day $\times 5$ days/week $\times 4$-week duration, while in Group B, ICH received MET for suboccipital muscles for 6 times/session/day $\times 5$ days/ week $\times 4$ weeks. Both the groups received the common intervention of stretching and strengthening for cervical muscles for 4 weeks. Pain scores (visual analog score [VAS]), disability (headache disability index [HD]), and cervical extension range of motion (ROM) were documented at baseline and $4^{\text {th }}$ week after intervention and analyzed.

Results: Group B demonstrated significance difference $(\mathrm{p}<0.05)$ in HDI, VAS, and cervical extension ROM when compared to Group A.

Conclusion: 4 week MET has the sufficient potential to decrease neck pain, disability, and increase cervical mobility among ICH as a non-surgical management.

Key words: Cervical pain, Cervicalgia, Cervicodynia, Physical therapy techniques, Range of motion.

(c) 2018 The Authors. Published by Innovare Academic Sciences Pvt Ltd. This is an open access article under the CC BY license (http://creativecommons. org/licenses/by/4. 0/) DOI: http://dx.doi.org/10.22159/ajpcr.2018.v11i9.26808

\section{INTRODUCTION}

Headache is one of the common problems which affect all the age groups among the global population. The International Headache Society has identified 14 different types of headache under two major categories, i.e. primary headache due to vascular or muscular lesion and secondary headache due to another source which includes inflammation or head and neck injury. The term cervicogenic headache was first coined by Norwegian Physician Dr. Ottar Sjaastad in the year 1983, and the term cervicogenic headache was recognized by the International Headache Society in the year 1988 as a secondary headache [1]. According to Sjaastad, cervicogenic headache is defined as the pain referred in one region of neck and it is perceived in one or more regions on face or neck $[2,3]$

Nearly $47 \%$ of global population suffer from headache [4], and of that, $15-20 \%$ is cervicogenic headache [5]. It is estimated that the prevalence of cervicogenic headache may vary from 0.4 to $4.6 \%$. In India, the cervicogenic headache patients were categorized as $43 \%$ urban, 57\% rural with $55.7 \%$ employed as handicraft workers, $28.3 \%$ as laborers, $10 \%$ as clerks, $4.9 \%$ as business executives, and $1.6 \%$ as health professionals [6]. The female population are commonly affected than the men population, and the female-to-male ratio will be $4: 1[7]$.

There are many treatment methods available for the management of individual with cervicogenic headache (ICH). The medical management includes cervical epidural corticosteroid injections, anesthetic nerve blocks, trigger point injections, and radiofrequency thermal neurolysis. Various surgical treatments such as neurotomy, dorsal rhizotomy and microvascular decompression of nerve roots are suggested to treat long-lasting cervicogenic headache in patients not responsive to any physical or drug therapy. [8].

Various surgical treatments such as neurotomy, dorsal rhizotomy and microvascular decompression of nerve roots are suggested to treat long-lasting cervicogenic headache in patients not responsive to any physical or drug therapy [9-17].

The effects of the above interventions are studied earlier yet a standard protocol or the intervention which effectively helps in pain, and disability reduction is inconclusive. This study is intended to compare the effects of two treatment techniques, namely MSNAGs and muscle energy technique, which are combined with common interventions like conventional exercises so that a better combination of treatment protocol can be adopted in ICH as non-surgical management.

\section{METHODS}

\section{Recruitment and allocation}

The study protocol was approved by the university research and ethics committee (ACS/2016/27), and the study was done strictly in accordance with the guidelines of Helsinki Declaration, revised 2013 [18]. A total of 30 ICHs were recruited by the simple random sampling (random number tables from standard statistics book) to participate in this twogroup pretest-posttest, single-blinded randomized clinical study. All the participants signed a written consent form before being subjected to therapy. Those who were not willing to sign the consent were excluded from the study. After the demographics, recruited ICH was randomly divided into two groups, Group A and Group B by block randomization. There were five blocks, with the matrix design of $6 \times 5$, where 6 being rows. Each block contained 6 chits (3 chits for each group), totaling 30 . 
The subjects were allotted to the group based on the randomly chosen chit. Once the block was allotted, next row block was opened. Thus, equal numbers of subjects were assigned to each group over time. Group A received MSNAG [17] for cervical spine and Group B received MET [16,19] for suboccipital muscles. Stretching and strengthening exercises for rectus capitis anterior, rectus capitis lateralis, longus colli, longus cervicis, semispinalis, splenius capitis and cervicis, scaleni, sternomastoitd, upper trapezius, pectoralis major and levator scapulae, rhomboids, and serratus anterior muscles for both the Groups A and B were given as a common intervention. Both the groups received the above-said intervention for 4 weeks period. The Consolidated Standards of Reporting Trials [20] flow chart describing the details of the study is displayed in Fig. 1.

\section{Mulligan's SNAGs in Group A}

MSNAG was given to the recruited ICH in Group A. The subject is seated, and the therapist stands behind the patient. The patient's head is cradled between the therapist body and his right arm. The right index, middle, and ring fingers wrap around the base of occiput, and the middle phalanx of little finger lies over the spinous process of $\mathrm{C} 2$ vertebra. The lateral border of the left thenar eminence lays over the right little finger. A gentle pressure is now applied by the therapist in a ventral direction on the spinous process of $\mathrm{C} 2$ vertebra while the skull remains neutral. This was quietly taken forward until end range is felt and this position was maintained for at least $10 \mathrm{~s}$. A total of 3 glides were given per session/day $\times 5$ days/week for 4 weeks' duration (Fig. 2).

\section{MET in Group B}

MET was received by Group B. The technique was administered for the suboccipital muscles, obliquus capitis superior and inferior, and rectus capitis posterior major and minor. The patient was positioned comfortably in supine lying position, and the therapist stands behind the patient. One hand of the therapist supports the patient's occiput, while the lateral border of index finger of the other hand is placed superior to the spinous process of the axis to stabilize it and anterior aspect of the therapist's shoulder rest on patient's forehead. The therapist applied a force to the occiput in a posterosuperior direction and asked the patient to poke the chin out and hold the position for $5 \mathrm{~s}$. This stretches the suboccipital muscles, and the subject is instructed to relax by poking the chin. This technique was given for 6 times/session/ day $\times 5$ days/week $\times 4$ weeks (Fig. 3 ).

\section{Common intervention of stretching and strengthening}

The stretching and the strengthening exercises were given to both Groups A and B as a common intervention. The exercises were given for rectus capitis anterior, rectus capitis lateralis, semispinalis, splenius capitis, splenius cervicis, longus colli, longus cervicis, sternomastoid, scalene, upper trapezius, levator scapulae and pectoralis major, rhomboids, and serratus anterior muscles. The duration of stretching was $15 \mathrm{~s}$ with 5 repetitions initially. Later, progression is made by increasing the duration from $30 \mathrm{~s}$ and repetition by 10 times. Strengthening exercises for the muscles initially begins with 10 repetitions maximum which are then finally progressed to 15 repetitions maximum per session.

\section{Outcome measures}

Both the groups were measured for their level of pain (visual analogue scale [VAS]), disability (headache disability index [HDI]), and cervical range of motion (ROM) (degree of extension) between Group A and Group B at baseline and $4^{\text {th }}$ week post-intervention.

\section{Data analysis}

The collected demographic and outcome measures were assessed for their normality using Shapiro-Wilk test. As the data follow normal distribution, all the descriptives were expressed in mean \pm standard deviation. Paired t-test was adopted to find the differences within Group A and Group B for pre-post intervention changes, while independent t-test was used to compare the changes in mean values of HDI, VAS, and cervical extension ROM between Group A and Group B at baseline and end of $4^{\text {th }}$ week intervention. The data were analyzed using statistical

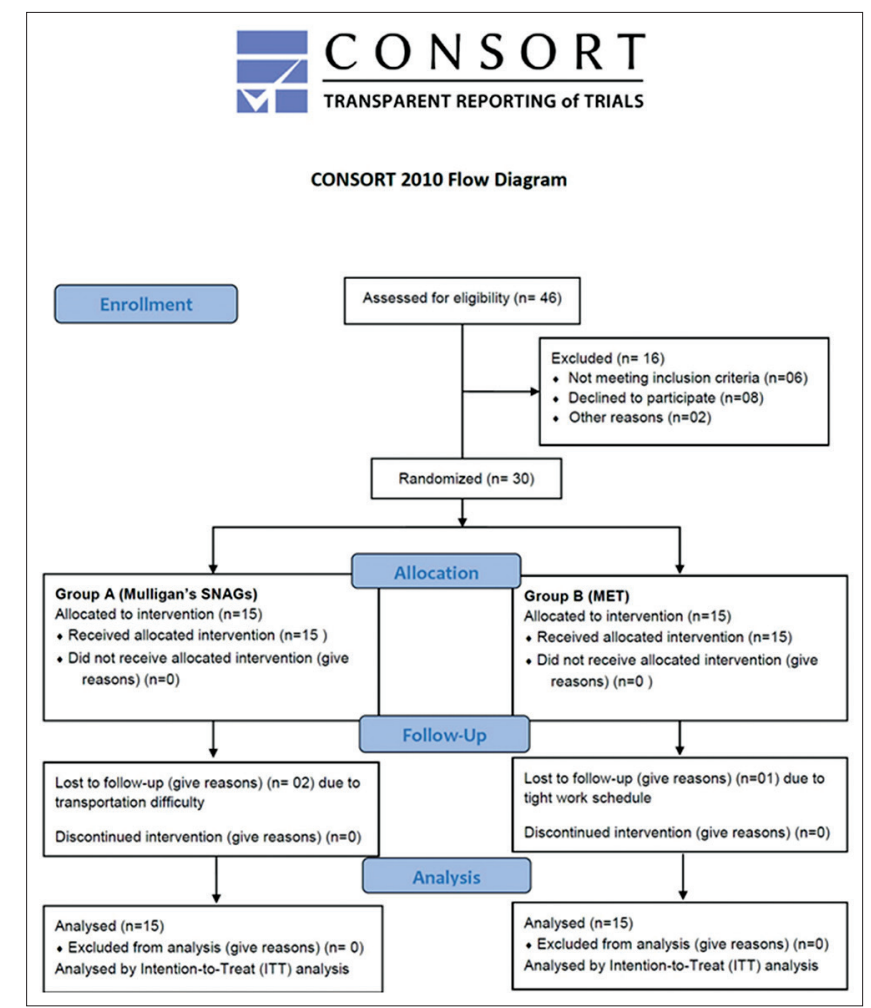

Fig. 1: Consort

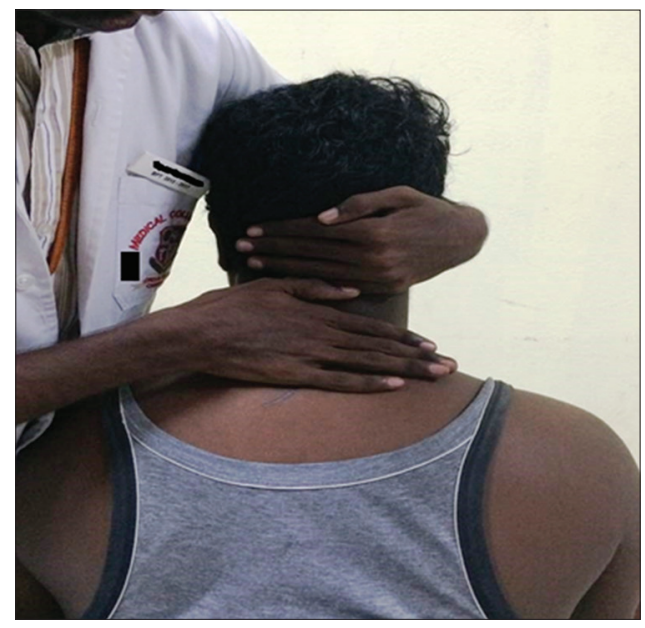

Fig. 2: Mulligan's SNAG's

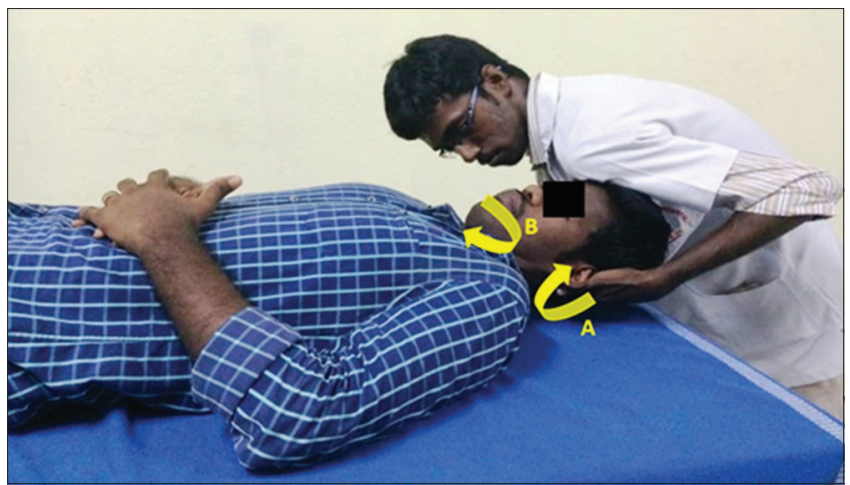

Fig. 3: Application of Muscle Energy Technique 
Table 1: Demographic characteristic of the individuals with cervicogenic headache recruited in Group A and Group B

\begin{tabular}{lll}
\hline Parameters & $\begin{array}{l}\text { Group A (male=3; } \\
\text { female=12) }\end{array}$ & $\begin{array}{l}\text { Group B (male=3; } \\
\text { female=12) }\end{array}$ \\
\hline $\mathrm{n}$ & 15 & 15 \\
Age (years) & $26.2 \pm 6.8$ & $25.7 \pm 7.1$ \\
Weight $(\mathrm{kg})$ & $62.4 \pm 7.5$ & $59.2 \pm 9.6$ \\
Height $(\mathrm{cm})$ & $168.2 \pm 9.2$ & $170.5 \pm 6.9$ \\
BMI $(\mathrm{kg} / \mathrm{m} 2)$ & $22.8 \pm 2.1$ & $22.3 \pm 2.9$ \\
\hline
\end{tabular}

BMI: Body mass index

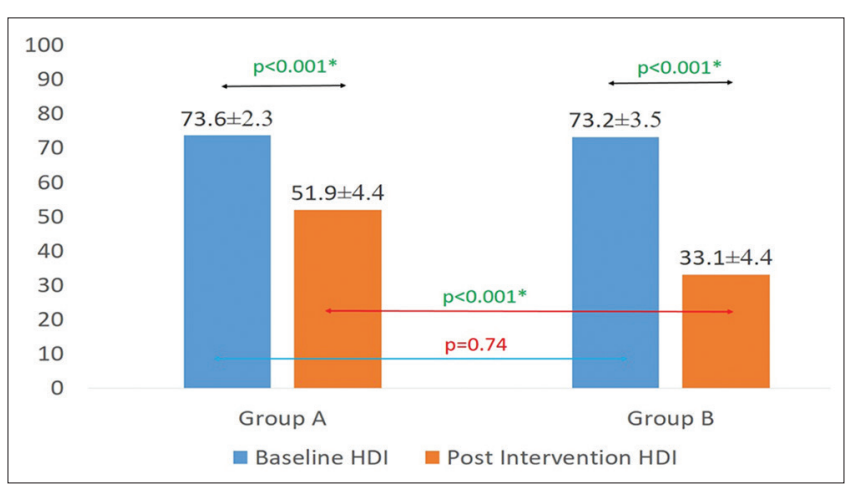

Graph 1: Headache disability index between Group A and Group B

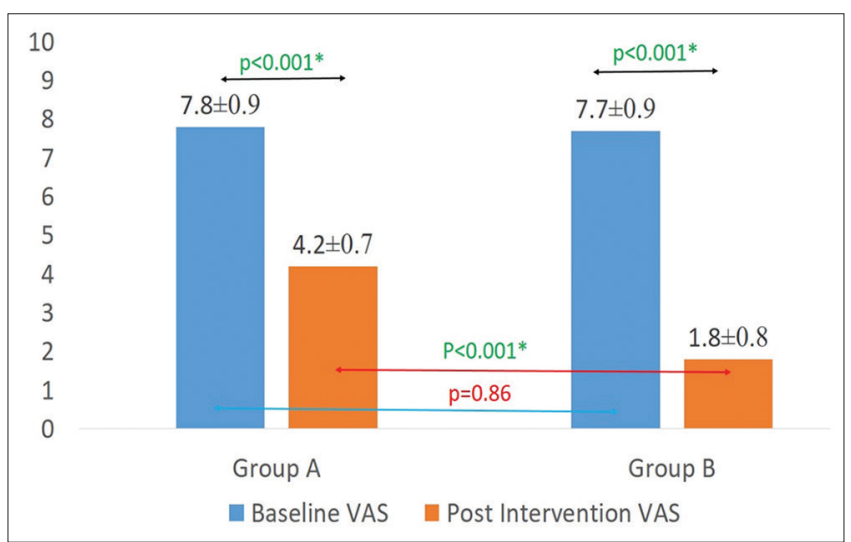

Graph 2: Visual analog score between Group A and Group B

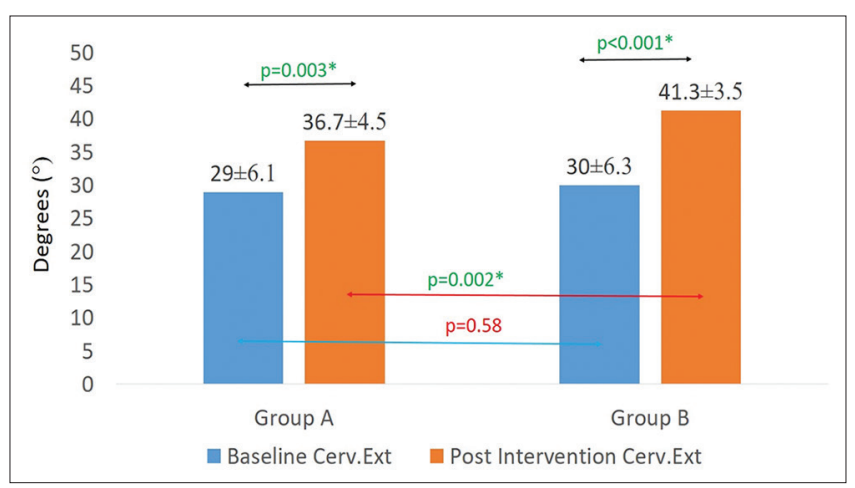

Graph 3: Cervical extension between Group A and Group B

software, Statistical Package for the Social Science (SPSS), IBM SPSS version 20.0 (Armonk, NY: IBM Corp.). The $\mathrm{p} \leq 0.05$ was considered to be statistically significant.

\section{RESULT}

Thirty subjects with cervicogenic headache were recruited for the study. Among them, 6 were male and remaining 24 were females. There exists no significant difference in the demographic characteristics between the two groups (Table 1). Both groups were compared at baseline and at the end of last exercise session for the following outcome measure, HDI (Graph 1), VAS (Graph 2), and Neck Extension ROM (Graph 3) were displayed. In all the outcome measures, Group B shows significant $(\mathrm{p}<0.05)$ improvement when compared to Group A.

\section{DISCUSSION}

The study was conducted on 30 subjects with cervicogenic headache. Group A was intervened with SNAG and Group B with MET, whereas stretching and strengthening exercises were given as a common intervention. Outcome measures included were Headache Disability Index scale, pain intensity by VAS, and cervical extension ROM by goniometer which was measured before the treatment and at the end of 4 weeks of treatment.

This study supports the findings of Hall et al. [17] demonstrating that SNAGs were effective in increasing the cervical ROM and also concluded the findings of Burns et al. that the application of muscle energy technique can produce acute increases in the active overall regional cervical ROM and significant reduction of pain and disability [16].

In MET, the neurological effects of loading of Golgi tendon organ of a skeletal muscle by an isometric contraction produce a post-isometric relaxation effect in the muscle. The pain is due to the muscle's inability to restore its anatomical length and joint restriction as a result of muscle tightness and shortening. Thus, MET physiologically relaxes the overactive muscle, thereby reducing the pain and improving the joint ROM $[21,22]$.

The application of SNAG influences correcting irregular position of articular elements and achieving correct biomechanics of the cervical spine. This technique causes a decrease in excessive reactivity of cervical nuclei of trigeminal nerve and blocks A-beta fibers stimuli that may result in pain relief and also limit the headache disability $[17,23]$. This study supports the findings of Patra et al. showing that Mulligan's SNAGs were an effective treatment in the management of cervicogenic headache [24].

There is a reduction of mean value of HDI, VAS, and improved cervical extension ROM in both the groups. However, the mean value of Group B post-test of HDI and VAS when compared with Group A had lesser mean value, implicating that MET (Group B) had potential to reduce disability and pain among ICH. This was even supported by increased cervical extension ROM in Group B. Hence, MET has higher potential to increase cervical mobility and thereby decrease the disability and pain among ICH.

\section{CONCLUSION}

Both MET and Mulligan's SNAGs have their role in the non-surgical management of cervicogenic headache. However, among both, MET has higher potential toward the management of ICH.

\section{AUTHOR'S CONTRIBUTIONS}

Veena Kirthika S - Project work and report writing. Padmanabhan K - Review of the article. Selvaraj Sudhakar - Data analysis and result. Vijaya Kumar M - Review of the article. 


\section{CONFLICTS OF INTEREST}

The authors do not have any conflicts of interest.

\section{REFERENCES}

1. Headache Classification Committee of the International Headache Society (IHS). The international classification of headache disorders, $3^{\text {rd }}$ edition (beta version). Cephalalgia 2013;33:629-808

2. Sjaastad O, Fredriksen TA, Pfaffenrath V. Cervicogenic headache: Diagnostic criteria. The cervicogenic headache international study group. Headache 1998;38:442-5.

3. Sjaastad O, Fredriksen TA, Pfaffenrath V. Cervicogenic headache: Diagnostic criteria. Headache 1990;30:725-6.

4. Stovner LJ, Hagen K, Jensen R, Katsarava Z, Lipton R, Scher A, et al. The global burden of headache: A documentation of headache prevalence and disability worldwide. Cephalalgia 2007;27:193-210.

5. Knackstedt H, Bansevicius D, Aaseth K, Grande RB, Lundqvist C, Russell MB, et al. Cervicogenic headache in the general population: The akershus study of chronic headache. Cephalalgia 2010;30:1468-76.

6. Shah PA, Nafee A. Clinical profile of headache and cranial neuralgias. J Assoc Physicians India 1999; 47:1072-5.

7. Haldeman S, Dagenais S. Cervicogenic headaches: A critical review. Spine J 2001;1:31-46.

8. Jansen J. Surgical treatment of non-responsive cervicogenic headache. Clin Exp Rheumatol 2000;18:S67-70.

9. Chaibi A, Russell MB. Manual therapies for cervicogenic headache: A systematic review. J Headache Pain 2012;13:351-9.

10. Fernandez-de-Las-Penas C, Cuadrado ML. Physical therapy for headaches. Cephalalgia 2015;36:1134-42.

11. Fernández-de-Las-Peñas C, Alonso-Blanco C, Cuadrado ML, Pareja JA. Spinal manipulative therapy in the management of cervicogenic headache. Headache 2005;45:1260-3.

12. Rana MV. Managing and treating headache of cervicogenic origin. Med Clin North Am 2013;97:267-80. Available from: http://www. sciencedirect.com/science/article/pii/S0025712512002027.

13. Racicki S, Gerwin S, Diclaudio S, Reinmann S, Donaldson M.
Conservative physical therapy management for the treatment of cervicogenic headache: A systematic review. J Man Manip Ther 2013;21:113-24.

14. Miller J, Gross A, D'Sylva J, Burnie SJ, Goldsmith CH, Graham N, et al. Manual therapy and exercise for neck pain: A systematic review. Man Ther 2010;15:334-54.

15. McDonnell MK, Sahrmann SA, Van Dillen L. A specific exercise program and modification of postural alignment for treatment of cervicogenic headache: A case report. J Orthop Sports Phys Ther 2005;35:3-15

16. Burns DK, Wells MR. Gross range of motion in the cervical spine: The effects of osteopathic muscle energy technique in asymptomatic subjects. J Am Osteopath Assoc 2006;106:137-42.

17. Hall T, Chan HT, Christensen L, Odenthal B, Wells C, Robinson K, et al. Efficacy of a C1-C2 self-sustained natural apophyseal glide (SNAG) in the management of cervicogenic headache. J Orthop Sports Phys Ther 2007;37:100-7.

18. World Health Organisation. Declaration of helsinki world medical association declaration of helsinki ethical principles for medical research involving human subjects. J Am Med Assoc 2013;310:2191-4.

19. Grimshaw DN. Cervicogenic headache: Manual and manipulative therapies. Curr Pain Headache Rep 2001;5:369-75.

20. Consort-Welcome to the CONSORT Website; 2010 Available from: http://www.consort-statement.org/. [Last cited on 2017 Aug 23].

21. Kalichman L, Ben David C. Effect of self-myofascial release on myofascial pain, muscle flexibility, and strength: A narrative review. J Bodyw Mov Ther 2017;21:446-51.

22. Zein-Hammoud M, Standley PR. Modeled osteopathic manipulative treatments: A Review of their in vitro effects on fibroblast tissue preparations. J Am Osteopath Assoc 2015;115:490-502.

23. Hearn A, Rivett DA. Cervical SNAGs: A biomechanical analysis. Man Ther 2002;7:71-9.

24. Patra RC, Mohanty P, Gautam AP. Effectiveness of C1-C2 sustained natural apophyseal glide combined with dry needling on pressure point threshold and headache disability in cervicogenic headache. Asian J Pharm Clin Red 2018;11:171-4. 